\title{
Ventricular fibrillation during electrical cardioversion of pre-excited atrial fibrillation
}

\author{
D Adlam, T Azeem
}

Postgrad Med J 2003;79:297-299

The Wolff-Parkinson-White syndrome can rarely present with pre-excited atrial fibrillation. In this condition the short refractory period of the accessory pathway can lead to rapid atrioventricular conduction. There is then a danger that at high heart rates the irregular broad complex tachycardia that results can deteriorate into ventricular fibrillation. The initial management of patients presenting in pre-excited atrial fibrillation requires cardioversion to sinus rhythm. This can be performed by DC cardioversion or pharmacological means. This paper describes the case of a patient presenting in pre-excited atrial fibrillation where electrical DC cardioversion lead to transient iatrogenic ventricular fibrillation.

T he Wolff-Parkinson-White syndrome is characterised by the occurrence of atrioventricular re-entry tachycardias in patients with the classical electrocardiographic features of a shortened PR interval and delta wave when in sinus rhythm. ${ }^{1}$ This results from the presence of an abnormal pathway for electrical conduction between atria and ventricles that is distinct from that of the atrioventricular node. If atrial fibrillation occurs in patients with Wolff-Parkinson-White syndrome, an accessory pathway with a short refractory period may allow rapid atrioventricular conduction. This leads to a fast, often irregular broad complex tachycardia termed pre-excited atrial fibrillation. ${ }^{2}$ Very rapid ventricular response rates can predispose to the development of ventricular fibrillation. ${ }^{3}{ }^{4}$ It is therefore important to achieve early cardioversion to sinus rhythm in patients presenting in pre-excited atrial fibrillation. This can be achieved by electrical DC cardioversion or pharmacologically with intravenous procainamide. Early referral is then recommended to a tertiary centre for electrophysiological studies with a view to pathway radiofrequency ablation. ${ }^{5}$ Verapamil shortens the effective refractory period of accessory pathway tissue. This may accelerate conduction through the accessory pathway increasing the risk of ventricular fibrillation. For this reason verapamil should be avoided in pre-excited atrial fibrillation. ${ }^{6}$

We present a case of a patient presenting in pre-excited atrial fibrillation who underwent electrical DC cardioversion but developed transient iatrogenic ventricular fibrillation before cardioverting to sinus rhythm.

\section{CASE REPORT}

A 30 year old man presented with sudden onset palpitations. This was associated with sweating and light headedness. He had no past history of palpitations and was otherwise fit and well. He was haemodynamically stable and clinical examination was normal but for the marked tachycardia.

The patient was transferred to the coronary care unit and a 12 lead electrocardiogram (ECG) was performed (fig 1). This showed a broad complex tachycardia with a variable R-R interval. Occasional narrow complex beats were also evident. A diagnosis of pre-excited atrial fibrillation was made and the clinical decision was taken to electrically cardiovert the patient under a brief general anaesthetic.

After lead attachment, the "synchronise" function of the defibrillator was activated. A single shock of 100 joules was administered (fig 2). This induced ventricular fibrillation. A further 200 joules DC shock was administered which successfully cardioverted the patient to sinus rhythm. The postcardioversion 12 lead ECG confirmed the classical features of WolffParkinson-White syndrome (fig 3).

The patient subsequently underwent electrophysiological studies with successful accessory pathway radiofrequency ablation and remains in sinus rhythm.

\section{DISCUSSION}

This case illustrates the typical ECG findings of a patient with Wolff-Parkinson-White syndrome both in sinus rhythm and pre-excited atrial fibrillation. Figure 3 clearly shows the short

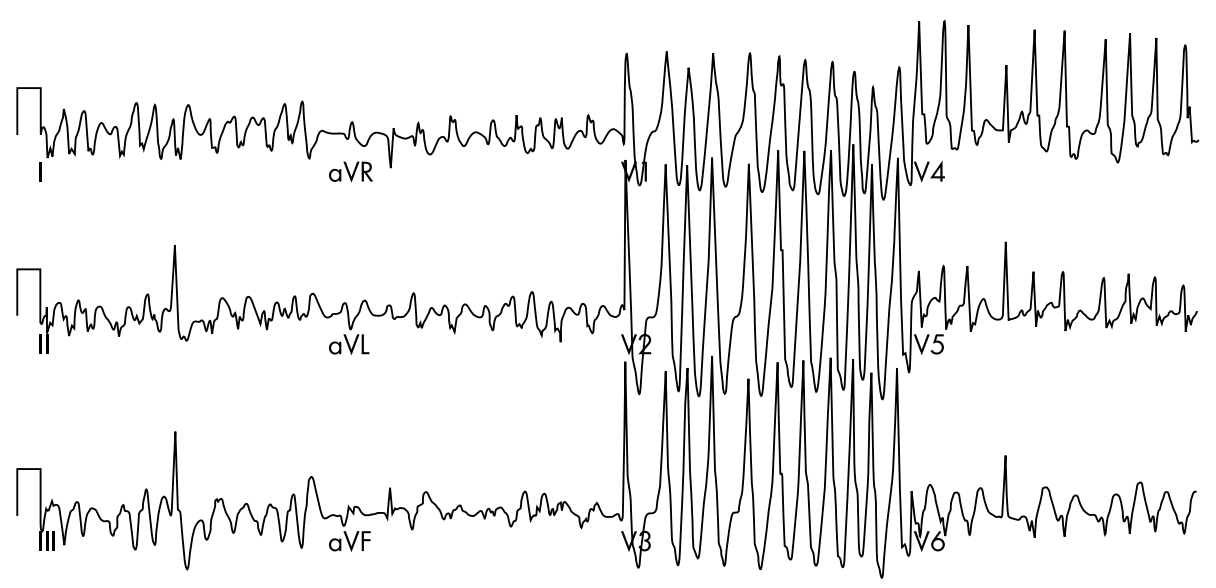

Figure 1 Admission 12 lead ECG. 

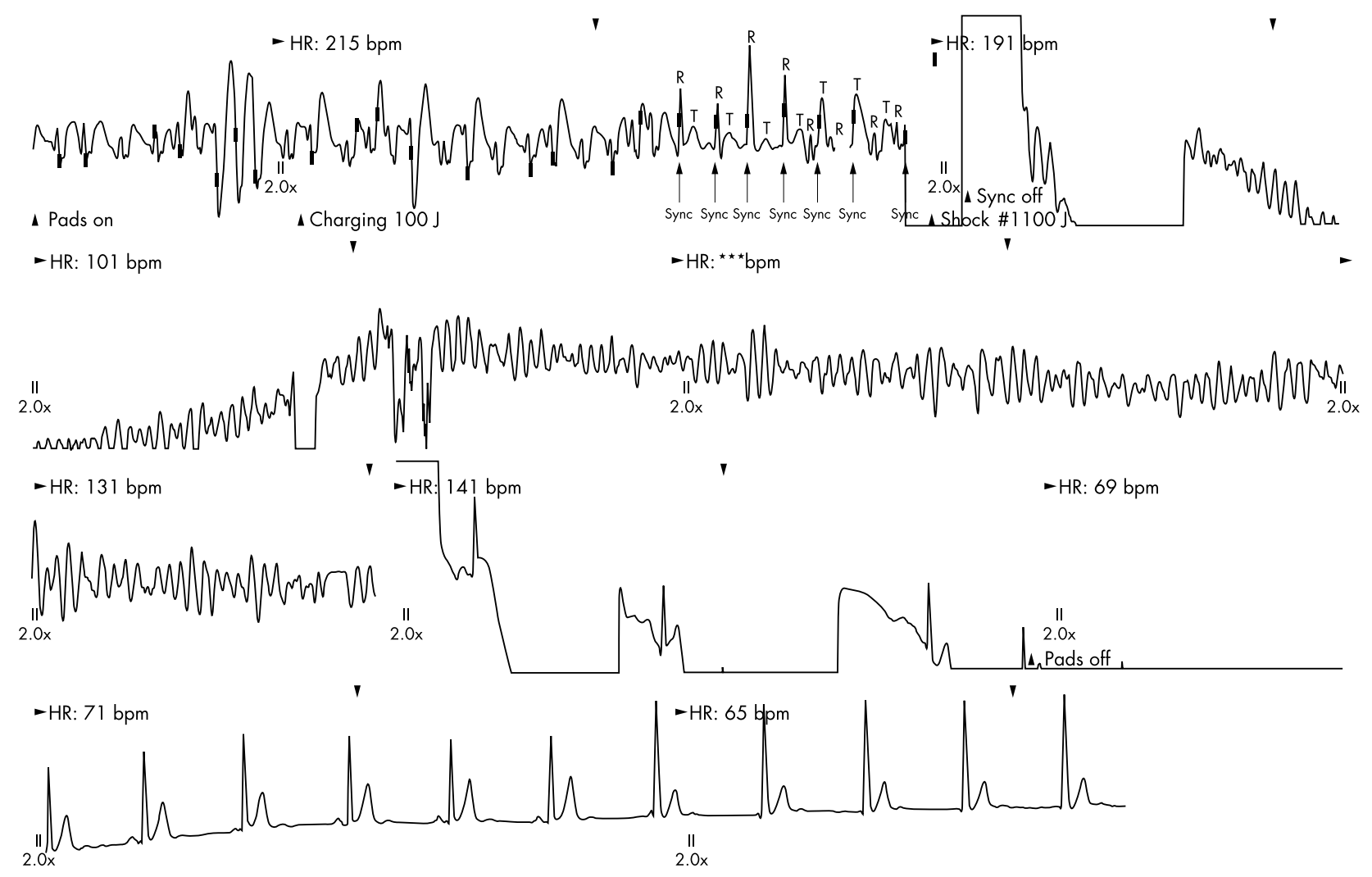

Figure 2 ECG rhythm strip during DC cardioversion.
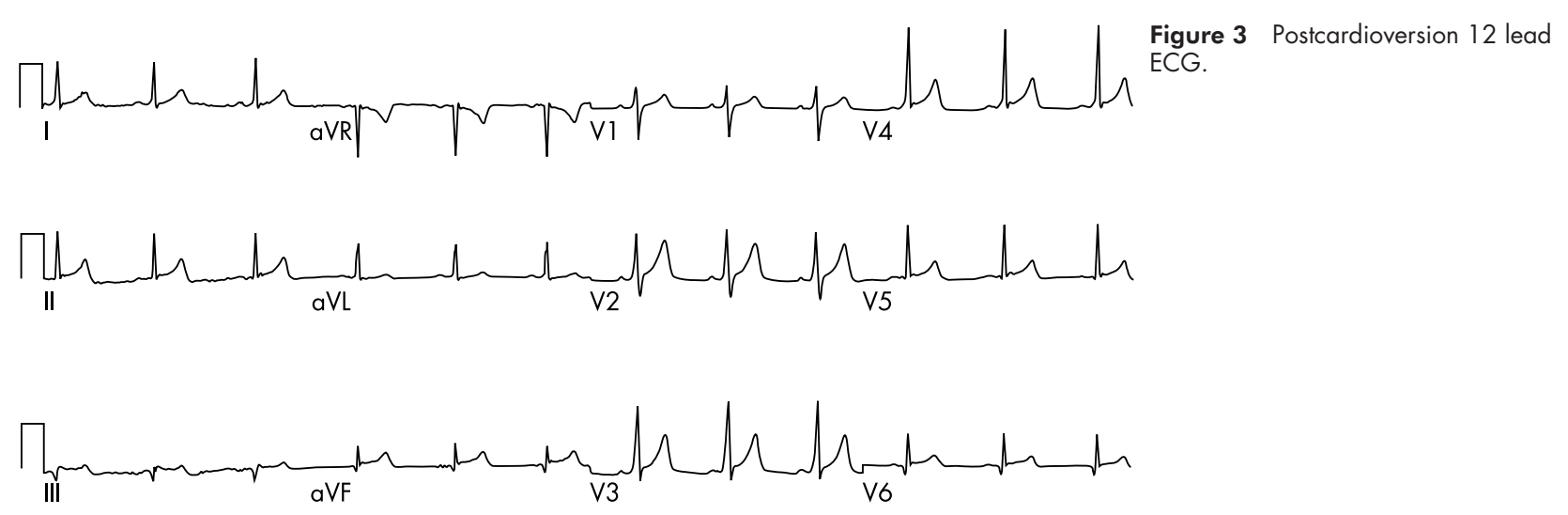

PR interval and delta wave (best seen in V4) classically described in Wolff-Parkinson-White syndrome. Figure 1 shows pre-excited atrial fibrillation. The tachycardia is broad complex but with a markedly irregular R-R interval suggestive of atrial fibrillation. The cardiac axis is completely different from the sinus ECG confirming accessory pathway conduction.

Although synchronised DC cardioversion is the safest way to treat these patients, this case also describes a cautionary tale for the use of DC cardioversion in pre-excited atrial fibrillation. In fig 2 the initial rhythm was pre-excited atrial fibrillation. The bold vertical markers indicate attempted synchronisation (also indicated by the vertical arrows labelled "Sync"). However as a result of the totally irregular rhythm and almost continuously changing QRS morphology, R-wave synchronisation was not consistent. It can be seen that the two last synchronisation bars and that associated with shock delivery occurred on $\mathrm{T}$ waves. The development of ventricular fibrillation that ensued was therefore a result of inappropriate synchronisation. The only previously reported case of this occurring resulted in the death of the patient from refractory ventricular fibrillation. ${ }^{7}$ Fortunately in this case delivery of a second DC shock led to a return to normal sinus rhythm.

\section{Learning points}

- Patients with the Wolff-Parkinson-White syndrome may present in pre-excited atrial fibrillation.

- Fast atrioventricular conduction via the accessory pathway during atrial fibrillation may predispose to the development of ventricular fibrillation. Urgent cardioversion to sinus rhythm is therefore needed.

- Great care should be taken during electrical DC cardioversion to ensure adequate $R$ wave synchronisation before shock delivery. Failure of synchronisation may lead to ventricular fibrillation

- All patients presenting in pre-excited atrial fibrillation should be referred for electrophysiological studies and pathway radiofrequency ablation. 
Patients with Wolff-Parkinson-White syndrome may rarely present in pre-excited atrial fibrillation. Great care should be taken during electrical cardioversion to ensure appropriate Rwave synchronisation. Once stabilised patients should be referred for electrophysiological studies and radiofrequency ablation of the accessory pathway.

\section{Authors' affiliations}

D Adlam, T Azeem, Department of Cardiology, Glenfield Hospital, Groby Road, Leicester LE3 9QP, UK

Correspondence to: Dr Adlam; davidadlam@doctors.org.uk

Submitted 13 October 2002

Accepted 10 December 2002

\section{REFERENCES}

1 Wolff L, Parkinson J, White PD. Bundle-branch block with short PR interval in healthy young people prone to paroxysmal tachycardia. Am Heart J 1930:5:685-704.

2 Campbell RWF, Smith RA, Gallagher JS, et al. Atrial fibrillation in the preexcitation syndrome. Am J Cardiol 1977;40:514-20.

3 Klein GJ, Bashore TM, Sellers TD, et al. Ventricular fibrillation in the Wolff-Parkinson-White syndrome. N Engl J Med 1979;301:1080-5.

4 Montoya PT, Brugada P, Smeets J, et al. Ventricular fibrillation in the Wolff- Parkinson-White syndrome. Eur Heart J 1991:12:144-50.

5 Ganz LI, Friedman PL. Medical progress: supraventricular tachycardia. N Engl J Med 1995;332:162-73.

6 Strasberg B, Sagie A, Rechavia E, et al. Deleterious effects of intravenous verapamil in Wolff-Parkinson-White patients and atrial fibrillation. Cardiovasc Drugs Ther 1989;2:801-6.

7 Ebrahimi R, Stanley AR. Electrical cardioversion resulting in death from synchronization failure. Am J Cardiol 1994;74:100-2.

\section{Readers' favourite}

\section{Top 10}

Click on the "Top 10" button on the homepage to see which are the best read articles each month

www.postgradmedj.com 